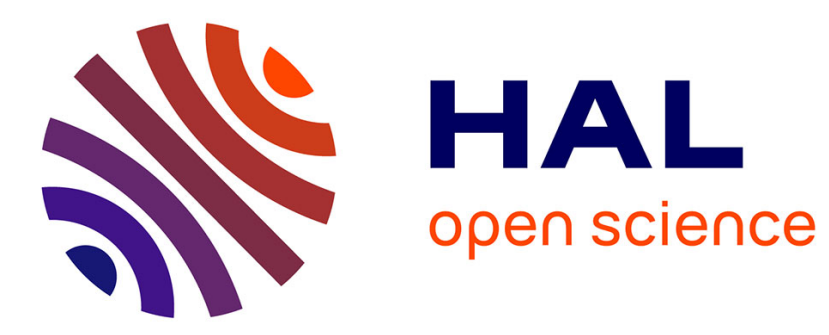

\title{
Asymptotic performance analysis of DOA finding algorithms with temporally correlated narrow-band signals
}

Jean-Pierre Delmas, Yann Meurisse

\section{- To cite this version:}

Jean-Pierre Delmas, Yann Meurisse. Asymptotic performance analysis of DOA finding algorithms with temporally correlated narrow-band signals. IEEE Transactions on Signal Processing, 2000. hal03435713

\section{HAL Id: hal-03435713 https://hal.science/hal-03435713}

Submitted on 18 Nov 2021

HAL is a multi-disciplinary open access archive for the deposit and dissemination of scientific research documents, whether they are published or not. The documents may come from teaching and research institutions in France or abroad, or from public or private research centers.
L'archive ouverte pluridisciplinaire HAL, est destinée au dépôt et à la diffusion de documents scientifiques de niveau recherche, publiés ou non, émanant des établissements d'enseignement et de recherche français ou étrangers, des laboratoires publics ou privés. 


\title{
Asymptotic performance analysis of DOA finding
}

\section{algorithms with temporally correlated narrow-band signals}

\author{
Jean-Pierre Delmas, Yann Meurisse *
}

November 16, 2021

\begin{abstract}
This correspondence focuses on the asymptotic performance analysis of general direction of arrival (DOA) finding algorithms under the stochastic model assumption in which source and noise signals are possibly non-Gaussian and possibly temporally correlated. We prove, in particular, that all the covariance-based DOA estimators are sensitive to the temporal correlation of the sources when the noise is temporally correlated; otherwise, most of them are insensitive to the temporal correlation of the sources except for the Toeplitzation and the augmentation techniques.
\end{abstract}

\section{Introduction}

Motivated by the popularity of the second-order algorithms in DOA estimation, many contributions have appeared that aim at establishing the asymptotic statistical performance of DOA estimators in the context of narrow-band array processing. These studies rest on different signal models. The deterministic and the stochastic model are the main models that have appeared in the literature. The deterministic model assumes the source signals fixed in all realizations and the noise to be a temporally uncorrelated Gaussian random process. In the stochastic model, the source and noise signals are generally assumed to be temporally uncorrelated Gaussian random processes. Many authors (see [1], [2], [3], [4] and the reference therein) compared the asymptotic performance of DOA algorithms with these two models and

${ }^{*}$ Institut National des Télécommunications. 9 rue Charles Fourier, 91011 Evry Cedex, France. tel: (33)-1-60 76 46 32. fax: (33)-1-60 7642 84. Email: delmas@int-evry.fr 
connected their performance to the Cramer-Rao bound. In fact, most DOA estimators have the same asymptotic statistical performance under these two models [3], [4] and with any distribution of the source signals in the stochastic model [5]. But all these contributions rely on the independence assumption of the successive snapshots. Consequently, performance analyses of these algorithms under mild assumptions remain of current interest.

It is the aim of this paper to investigate the performance of DOA estimators under the general stochastic model assumption in which both the source and noise signals are possibly temporally correlated and possibly non-Gaussian random processes. Ordinarily, the performance analysis of these second-order algorithms relies on the distribution of the empirical spatial covariance matrix $\mathbf{R}_{x}(n) \stackrel{\text { def }}{=} \frac{1}{n} \sum_{t=1}^{n} \mathbf{x}_{t} \mathbf{x}_{t}^{H}$. These studies use two approaches. The first one is based on perturbation calculus induced by its complex Wishart distribution when the snapshots $\mathbf{x}_{t}$ are Gaussian. The second is based on a continuity theorem (e.g. [6, theorem, p. 122]) which transfers the asymptotic normality issued from its complex asymptotic Gaussian distribution derived from the classical central limit theorem to any regular function of this covariance. When the snapshots $\mathbf{x}_{t}$ are not independent, the distribution of $\mathbf{R}_{x}(n)$ is not complex Wishart in the Gaussian case for the first approach and the classical central limit theorem cannot be applied for the second approach. We adopt in this paper the general functional method of [7], in which the Gaussian asymptotic distribution of the covariance-based DOA estimates is derived from the Gaussian asymptotic distribution of the empirical covariance matrix. This allows us to give closed-form expressions for the asymptotic covariance matrices of DOA estimates and to specify the conditions for which these expressions are sensitive to the distribution and the temporal correlation of the sources.

This correspondence is organized as follows. The model of dependent snapshots $\mathbf{x}_{t}$ is defined in Section 2. Then in Section 3, the asymptotic normality of $\mathbf{R}_{x}(n)$ is established for these models of dependence where a central limit theorem is given. A general functional approach providing a common unifying framework for asymptotic DOA estimation performance analysis is presented in Section 4. The case of uniform linear or rectangular arrays with the Toeplitzation and augmentation techniques are addressed. Finally, in Section 5 some simulations are presented. 


\section{Signal model}

Let an arbitrary array composed of $M$ sensors receive $K$ narrow-band waves. These narrow-band signals are assumed to have a common center frequency and are in the same bandwidth $B$. Let $u_{t, k}, \boldsymbol{\Theta}_{k}$ and $\mathbf{e}\left(\boldsymbol{\Theta}_{k}\right)$ denote, respectively for the source $k$, the complex envelope of the emitted signal by this source at time $t$, the unknown spatial parameters which are referred to as the DOA and the so-called steering vector of this source. The $M$-vector of the observed complex envelopes of the sensor outputs is typically modeled by

$$
\mathbf{x}_{t}=\sum_{k=1}^{K} u_{t, k} \mathbf{e}\left(\boldsymbol{\Theta}_{k}\right)+\mathbf{v}_{t} .
$$

Here, $\mathbf{v}_{t}$ represents the $M$-vector of observed complex envelope of sensor output additive noise at time $t$. Ordinarily several independent measurements $\mathbf{x}_{t}$ are made by sampling the complex envelopes at times $t$ such that $\left(u_{t, k}, \mathbf{v}_{t}\right)_{t=1, \ldots, n}$ are independent. We suppose in this paper that the complex envelopes of the sensor outputs are uniformly sampled at a frequency greater than or equal to $B$. As a consequence the observations $\left(\mathbf{x}_{t}\right)_{t=1, \ldots, n}$ are no longer independent. $\mathbf{v}_{t}$ and $\left(u_{t, k}\right)_{t=1, \ldots, n}$ are modeled as zero-mean with finite fourth order moments not necessarily Gaussian stationary random processes. $\mathbf{v}_{t}$ is supposed independent of $\left(u_{t, k}\right)_{t=1, \ldots, n}$. The spatial covariance matrix $\mathbf{R}_{x} \stackrel{\text { def }}{=} \mathrm{E}\left(\mathbf{x}_{t} \mathbf{x}_{t}^{H}\right)$ reads:

$$
\mathbf{R}_{x}=\mathbf{E}(\boldsymbol{\Theta}) \mathbf{R}_{u} \mathbf{E}^{H}(\boldsymbol{\Theta})+\sigma_{v}^{2} \mathbf{C}
$$

with $\mathbf{E}(\boldsymbol{\Theta}) \stackrel{\text { def }}{=}\left[\mathbf{e}\left(\boldsymbol{\Theta}_{1}\right), \ldots, \mathbf{e}\left(\boldsymbol{\Theta}_{K}\right)\right], \mathbf{R}_{u} \stackrel{\text { def }}{=} \mathrm{E}\left(\mathbf{u}_{t} \mathbf{u}_{t}^{H}\right)$ where $\mathbf{u}_{t} \stackrel{\text { def }}{=}\left(u_{t, 1}, \ldots, u_{t, K}\right)^{T}$ and $\mathrm{E}\left(\mathbf{v}_{t} \mathbf{v}_{t}^{H}\right)=\mathbf{R}_{v}=$ $\sigma_{v}^{2} \mathbf{C}$ where $\mathbf{C}$ is a known positive definite matrix. In order to consider the asymptotic distribution of the estimated spatial covariance matrix $\mathbf{R}_{x}(n)$, we consider for simplicity that $u_{t, k}$ are either harmonic random processes $\left(u_{t, k}=\sum_{l=1}^{L_{k}} a_{k, l} e^{i \alpha_{k, l}} e^{i 2 \pi f_{k, l} t}\right.$, where $\left(f_{k, l}\right)_{k=1, \ldots, K, l=1, \ldots, L_{k}}$ are fixed distinct positive real numbers in $]-1 / 2,+1 / 2\left[, a_{k, l}\right.$ are fixed positive real numbers and $\alpha_{k, l}$ are random variables uniformly distributed in $[0,2 \pi]$ and mutually independent), or complex ARMA processes with power $\sigma_{k}^{2}$, power spectral density $S_{u_{k}}(f)$ and power cross-spectral density $K \times K$-matrix $\mathbf{S}_{u}(f)$. If the fourth order polyspectrum of the $K$ sources $u_{t, k}$ for $k_{1}, k_{2}, k_{3}, k_{4}=1, \ldots, K$ is defined as

$$
\begin{gathered}
\rho_{k_{1}, k_{2}, k_{3}, k_{4}}\left(f, f^{\prime}, f^{\prime \prime}\right) \stackrel{\text { def }}{=} \sum_{\tau, \tau^{\prime}, \tau^{\prime \prime}} \operatorname{Cum}\left(u_{0, k_{1}}, u_{\tau, k_{2}}^{*}, u_{\tau^{\prime}, k_{3}}, u_{\tau^{\prime \prime}, k_{4}}^{*}\right) e^{i 2 \pi\left(f \tau+f^{\prime} \tau^{\prime}+f^{\prime \prime} \tau^{\prime \prime}\right)}, \\
{\left[\mathbf{Q}_{u}\right]_{K(j-1)+i, K(l-1)+k}=\int_{-1 / 2}^{+1 / 2} \int_{-1 / 2}^{+1 / 2} \rho_{i, j, l, k}\left(f, f^{\prime},-f^{\prime}\right) d f d f^{\prime}}
\end{gathered}
$$


denotes the $K^{2} \times K^{2}$ fourth order cumulant matrix. The same ARMA assumption and notations are adopted to the $M$-variate $\mathbf{v}_{t}$.

Usually, in the context of narrow-band waves, the observations $\left(\mathbf{x}_{t}\right)_{t=1, \ldots, n}$ are assumed zero-mean circular Gaussian and independent. So the ensemble average $\mathbf{R}_{x}(n)$ is a sufficient statistic and consequently all direction-finding algorithms are based on $\mathbf{R}_{x}(n)$. In this context, all the asymptotic performance analyses are based on the distribution of $\sum_{t=1}^{n} \mathbf{x}_{t} \mathbf{x}_{t}^{H}$, i.e., a central complex Wishart distribution. In this paper, we go on using second-order direction-finding algorithms. But, as in our signal model the observations $\left(\mathbf{x}_{t}\right)_{t=1, \ldots, n}$ are no longer independent, all the results based on this Wishart distribution are not usable. We need to know the asymptotic distribution of $\mathbf{R}_{x}(n)$.

\section{Spatial covariance matrix}

Covariance-based DOA estimators will turn out to be asymptotically normal as the number $n$ of observations goes to infinity. In this section, we focus on a central limit theorem to be used for establishing DOA asymptotic normality in the next section. We show that the asymptotic distribution of the spatial covariance matrix $\mathbf{R}_{x}(n)$ is very sensitive to the model of dependence between snapshots. We prove the following theorem:

Theorem $1 \sqrt{n}\left(\operatorname{Vec}\left(\mathbf{R}_{x}(n)\right)-\operatorname{Vec}\left(\mathbf{R}_{x}\right)\right)$ converges in distribution to the zero-mean complex Gaussian distribution of covariance $\mathbf{C}_{R_{x}}$

$$
\sqrt{n}\left(\operatorname{Vec}\left(\mathbf{R}_{x}(n)\right)-\operatorname{Vec}\left(\mathbf{R}_{x}\right)\right) \stackrel{\mathcal{L}}{\rightarrow} \mathcal{N}\left(\mathbf{0}, \mathbf{C}_{R_{x}}, \mathbf{C}_{R_{x}} \mathbf{K}\right) .
$$

Furthermore $\mathrm{E}\left(\mathbf{R}_{x}(n)\right)=\mathbf{R}_{x}$ and

$$
\lim _{n \rightarrow \infty} n \operatorname{Cov}\left(\operatorname{Vec}\left(\mathbf{R}_{x}(n)\right)\right)^{1}=\mathbf{C}_{R_{x}}
$$

where $\mathbf{C}_{R_{x}}$ reads:

$$
\begin{aligned}
\mathbf{C}_{R_{x}}= & \left(\mathbf{E}(\boldsymbol{\Theta}) \otimes_{c} \mathbf{E}(\boldsymbol{\Theta})\right) \mathbf{C}_{R_{u}}\left(\mathbf{E}^{H}(\boldsymbol{\Theta}) \otimes_{c} \mathbf{E}^{H}(\boldsymbol{\Theta})\right)+\mathbf{C}_{R_{v}} \\
& +\left(\mathbf{E}(\boldsymbol{\Theta}) \otimes_{c} \mathbf{I}_{M}\right) \mathbf{C}_{R_{u, v}}\left(\mathbf{E}^{H}(\boldsymbol{\Theta}) \otimes_{c} \mathbf{I}_{M}\right)+\left(\mathbf{I}_{M} \otimes_{c} \mathbf{E}(\boldsymbol{\Theta})\right) \mathbf{C}_{R_{v, u}}\left(\mathbf{I}_{M} \otimes_{c} \mathbf{E}^{H}(\boldsymbol{\Theta})\right)
\end{aligned}
$$

\footnotetext{
${ }^{1} \operatorname{Cov}\left(\operatorname{Vec}\left(\mathbf{R}_{x}(n)\right)\right)$ denotes $\mathrm{E}\left(\operatorname{Vec}\left(\mathbf{R}_{x}(n)-\mathbf{R}_{x}\right) \operatorname{Vec}^{H}\left(\mathbf{R}_{x}(n)-\mathbf{R}_{x}\right)\right)$. We note that $\operatorname{Vec}^{T}\left(\mathbf{R}_{x}(n)-\mathbf{R}_{x}\right)=\operatorname{Vec}^{H}\left(\mathbf{R}_{x}(n)-\right.$ $\left.\mathbf{R}_{x}\right) \mathbf{K}$, so $\mathrm{E}\left(\operatorname{Vec}\left(\mathbf{R}_{x}(n)-\mathbf{R}_{x}\right) \operatorname{Vec}^{T}\left(\mathbf{R}_{x}(n)-\mathbf{R}_{x}\right)\right)=\mathrm{E}\left(\operatorname{Vec}\left(\mathbf{R}_{x}(n)-\mathbf{R}_{x}\right) \operatorname{Vec}^{H}\left(\mathbf{R}_{x}(n)-\mathbf{R}_{x}\right)\right) \mathbf{K}$. Therefore the noncircular complex Gaussian asymptotic distribution of $\mathbf{R}_{x}(n)$ is characterized by $\mathbf{C}_{R_{x}}$ only.
} 
with $\mathbf{C}_{R_{u}}=\int_{-1 / 2}^{+1 / 2} \mathbf{S}_{u}(f) \otimes_{c} \mathbf{S}_{u}(f) d f+\mathbf{Q}_{u}$ in the $A R M A$ case and $\mathbf{C}_{R_{u}}=\mathbf{O}$ in the harmonic case, $\mathbf{C}_{R_{v}}=\int_{-1 / 2}^{+1 / 2} \mathbf{S}_{v}(f) \otimes_{c} \mathbf{S}_{v}(f) d f+\mathbf{Q}_{v}, \mathbf{C}_{R_{u, v}}=\sum_{n=-\infty}^{+\infty} \mathbf{R}_{u}^{n} \otimes_{c} \mathbf{R}_{v}^{n}, \mathbf{C}_{R_{v, u}}=\sum_{n=-\infty}^{+\infty} \mathbf{R}_{v}^{n} \otimes_{c} \mathbf{R}_{u}^{n}$, where $\mathbf{R}_{u}^{n} \stackrel{\text { def }}{=} \mathrm{E}\left(\mathbf{u}_{t} \mathbf{u}_{t-n}^{H}\right)$ and $\mathbf{R}_{v}^{n} \stackrel{\text { def }}{=} \mathrm{E}\left(\mathbf{v}_{t} \mathbf{v}_{t-n}^{H}\right) . \mathbf{A} \otimes_{c} \mathbf{B}$ denotes the block matrix, the $(i, j)$ block element of which is $b_{i, j}^{*} \mathbf{A}$ and $\mathbf{K}$ is the vec-permutation matrix defined by $\operatorname{Vec}\left(\mathbf{A}^{T}\right)=\mathbf{K V e c}(\mathbf{A})$ for any square matrix A.

In (3.1) a complex random $p \times 1$ vector $\mathbf{y}$ has a zero-mean complex Gaussian distribution specified by $p \times p$ positive definite matrix $\boldsymbol{\Sigma}_{1}$ and $p \times p$ symmetric matrix $\boldsymbol{\Sigma}_{2}$ and denoted $\mathcal{N}\left(\mathbf{0}, \boldsymbol{\Sigma}_{1}, \boldsymbol{\Sigma}_{2}\right)$ if the $2 p$-joint distribution of the real and imaginary part of $\mathbf{y}$ is $2 p$-zero-mean Gaussian, i.e. for any complex $p \times 1$ vector $\mathbf{w}$ : the real scalar $\mathbf{w}^{H} \mathbf{y}+\left(\mathbf{w}^{H} \mathbf{y}\right)^{H}$ has a zero-mean Gaussian distribution with variance $2 \mathbf{w}^{H} \boldsymbol{\Sigma}_{1} \mathbf{w}+\mathbf{w}^{H} \boldsymbol{\Sigma}_{2} \mathbf{w}^{*}+\mathbf{w}^{T} \boldsymbol{\Sigma}_{2}^{*} \mathbf{w}$ where $\mathrm{E}\left(\mathbf{y} \mathbf{y}^{H}\right)=\boldsymbol{\Sigma}_{1}$ and $\mathrm{E}\left(\mathbf{y} \mathbf{y}^{T}\right)=\boldsymbol{\Sigma}_{2}$.

(3.2) is proved after straightforward but tedious algebric manipulations. Then, to prove (3.1), we adapt the steps of $([8$, section 7.3$])$ to our problem.

\section{Asymptotic distribution of DOA estimates}

\subsection{Functional approach}

To consider the asymptotic performance of a covariance-based DOA algorithm, we adopt a functional analysis $^{2}$ which consists in recognizing that the whole process of constructing an estimate $\boldsymbol{\Theta}(n)$ of $\boldsymbol{\Theta}$ is equivalent to defining a functional relation linking this estimate $\boldsymbol{\Theta}(n)$ to the statistics $\mathbf{R}_{x}(n)$ from which it is inferred. This functional dependence is denoted $\boldsymbol{\Theta}(n)=\operatorname{alg}\left(\mathbf{R}_{x}(n)\right)$. Clearly, $\boldsymbol{\Theta}=\operatorname{alg}\left(\mathbf{R}_{x}\right)$, so the different algorithms $\operatorname{alg}($.$) constitute distinct extensions of the mapping \mathbf{R}_{x} \rightarrow \boldsymbol{\Theta}$ generated by any unstructured Hermitian matrix $\mathbf{R}_{x}(n)$. In the following, we consider "regular" algorithms. More specifically, we assume the conditions given in [5]:

1. The function $\operatorname{alg}($.$) is differentiable in a neighborhood of \mathbf{R}_{x}$, i.e., if $\mathbf{D}_{\Theta, \mathbf{R}_{x}}^{\text {alg }}$ denotes the $K \times M^{2}$ matrix of this differential evaluated at point $\mathbf{R}_{x}$

$$
\operatorname{alg}\left(\mathbf{R}_{x}+\delta \mathbf{R}\right)=\mathbf{\Theta}+\mathbf{D}_{\boldsymbol{\Theta}, \mathbf{R}_{x}}^{\mathrm{alg}} \operatorname{Vec}(\delta \mathbf{R})+o(\delta \mathbf{R}) .
$$

\footnotetext{
${ }^{2}$ A similar approach based on the implicit function theorem was introduced by Xu and Kaveh [9].
} 
2. For any $\boldsymbol{\Theta}$ and any positive definite source correlation matrix $\mathbf{R}_{u}$ (condition 2a) or for any $\boldsymbol{\Theta}$ and any positive definite diagonal source correlation matrix $\mathbf{R}_{u}$ (condition $2 \mathrm{~b}$ )

$$
\operatorname{alg}\left(\mathbf{E}(\boldsymbol{\Theta}) \mathbf{R}_{u} \mathbf{E}^{H}(\boldsymbol{\Theta})+\sigma_{v}^{2} \mathbf{C}\right)=\mathbf{\Theta}
$$

Under appropriate hypotheses on the array manifold, the requirements 1 and $2 \mathrm{a}$ are met by most of the second-order DOA estimators. The requirements 1 and $2 \mathrm{~b}$ are met by the second-order DOA estimators that suppose the sources spatially uncorrelated. The following lemma (proved under conditions 1 and $2 \mathrm{a}$ in [5]) is used in next section, to prove the invariance of the asymptotic distribution of the DOA's with respect to the distribution and the temporal correlation of the sources.

Lemma 1 Under conditions 1 and $2 a$ [resp. conditions 1 and 2b], one has the constraints upon $\mathbf{D}_{\boldsymbol{\Theta}, \mathbf{R}_{x}}^{\text {alg }}$

$$
\mathbf{D}_{\boldsymbol{\Theta}, \mathbf{R}_{x}}^{\mathrm{alg}}\left(\mathbf{E}(\boldsymbol{\Theta}) \otimes_{c} \mathbf{E}(\boldsymbol{\Theta})\right)=\mathbf{O} \quad\left[\text { resp., } \mathbf{D}_{\boldsymbol{\Theta}, \mathbf{R}_{x}}^{\mathrm{alg}}\left(\mathbf{e}\left(\boldsymbol{\Theta}_{k}\right) \otimes_{c} \mathbf{e}\left(\boldsymbol{\Theta}_{k}\right)\right)=\mathbf{0}, \quad k=1, \ldots, K\right]
$$

\subsection{Standard algorithms}

By the regularity condition (4.1), the asymptotic behaviors of $\boldsymbol{\Theta}(n)$ and $\mathbf{R}_{x}(n)$ are directly related. The standard result on regular functions of asymptotically normal statistics (see. e.g. [6, theorem, p. 122]) applies:

$$
\sqrt{n}(\boldsymbol{\Theta}(n)-\boldsymbol{\Theta}) \stackrel{\mathcal{L}}{\rightarrow} \mathcal{N}\left(\mathbf{0}, \mathbf{C}_{\Theta}\right)
$$

with

$$
\mathbf{C}_{\Theta}=\lim _{n \rightarrow \infty} n \mathrm{E}\left((\boldsymbol{\Theta}(n)-\boldsymbol{\Theta})(\boldsymbol{\Theta}(n)-\boldsymbol{\Theta})^{T}\right)=\mathbf{D}_{\boldsymbol{\Theta}, \mathbf{R}_{x}}^{\mathrm{alg}} \mathbf{C}_{R_{x}}\left(\mathbf{D}_{\boldsymbol{\Theta}, \mathbf{R}_{x}}^{\mathrm{alg}}\right)^{H}
$$

We can now state our main result:

Theorem 2 For Gaussian or non-Gaussian, ARMA or harmonic source signals, the asymptotic covariance of any covariance-based DOA estimators that do not require the sources spatially uncorrelated, has the common closed-form expression when the noise $\mathbf{v}_{t}$ is temporally uncorrelated:

$$
\mathbf{C}_{\Theta}=\mathbf{D}_{\boldsymbol{\Theta}, \mathbf{R}_{x}}^{\mathrm{alg}}\left(\mathbf{E}(\boldsymbol{\Theta}) \mathbf{R}_{u} \mathbf{E}^{H}(\boldsymbol{\Theta}) \otimes_{c} \sigma_{v}^{2} \mathbf{C}+\sigma_{v}^{2} \mathbf{C} \otimes_{c} \mathbf{E}(\boldsymbol{\Theta}) \mathbf{R}_{u} \mathbf{E}^{H}(\boldsymbol{\Theta})+\sigma_{v}^{4} \mathbf{C} \otimes_{c} \mathbf{C}+\mathbf{Q}_{v}\right)\left(\mathbf{D}_{\Theta, \mathbf{R}_{x}}^{\mathrm{alg}}\right)^{H}
$$

Proof: In this situation $\mathbf{R}_{v}^{n}=\delta_{0, n} \sigma_{v}^{2} \mathbf{C}$. The closed-form expression follows by application of (3.3), (4.5) and the first part of (4.3). 
This result extends the result in [5]. We note that if the noise is temporally correlated, the terms $\mathbf{C}_{R_{u, v}}$ and $\mathbf{C}_{R_{v, u}}$ of (3.3) do not reduce to the spatial terms $\mathbf{R}_{u} \otimes_{c} \mathbf{R}_{v}$ and $\mathbf{R}_{v} \otimes_{c} \mathbf{R}_{u}$ and so the performance of all the covariance-based DOA algorithms are sensitive to the temporal correlation of the sources when the noise is temporally correlated. In the next subsections, we show that the asymptotic performance of DOA algorithms that require the sources spatially uncorrelated are sensitive to the distribution and the coloration of the spectrum of the sources, even when the noise is temporally uncorrelated.

\subsection{Toeplitzation techniques}

For $M$-uniform linear array (ULA) [resp., $M_{1} \times M_{2}$-uniform rectangular array (URA)], spatially uncorrelated sources and spatially white noise, $\mathbf{R}_{x}$ exhibits a Toeplitz [resp., Toeplitz, block-Toeplitz] structure. The estimated spatial covariance matrix $\mathbf{R}_{x}(n)$ accuracy is significantly improved by averaging along its diagonals [resp., its subblock diagonals]. The resulting estimate $\mathbf{R}_{x}^{\text {to }}(n)$ is referred to as the "Toeplitzed" estimated spatial covariance matrix. Because this "Toeplitzation", also known as redundancy averaging [10], operates a linear transform on $\mathbf{R}_{x}(n)$ thanks to the "Toeplitzation" projection matrix $\mathbf{A}_{\text {to }}$ $\left(\operatorname{Vec}\left(\mathbf{R}_{x}^{\text {to }}(n)\right)=\mathbf{A}_{\text {to }} \operatorname{Vec}\left(\mathbf{R}_{x}(n)\right)\right)$, Theorem 1 is extending to $\mathbf{R}_{x}^{\text {to }}(n)$ with the asymptotic covariance matrix $\mathbf{C}_{R_{x}}^{\text {to }}=\mathbf{A}_{\text {to }} \mathbf{C}_{R_{x}} \mathbf{A}_{\text {to }}$. By the regularity condition (4.1) of subsection 4.1, the estimated DOA's are asymptotically normal with asymptotic covariance:

$$
\mathbf{C}_{\Theta}^{\text {to }}=\mathbf{D}_{\Theta, \mathbf{R}_{x}}^{\mathrm{alg}} \mathbf{A}_{\text {to }} \mathbf{C}_{R_{x}} \mathbf{A}_{\text {to }}\left(\mathbf{D}_{\Theta, \mathbf{R}_{x}}^{\mathrm{alg}}\right)^{H}
$$

In contrast to the classical covariance-based DOA algorithms, we show in the following that the Toeplitzed covariance-based DOA algorithms are sensitive to the spectral shape of the spectrum of the sources. More precisely, the following properties are proved: ${ }^{3}$

1. The Toeplitzation is not sensitive to the distribution of the ARMA sources if the sources are not only spatially uncorrelated but are independent.

Proof: If the sources are independent, the only nonzero terms of the fourth-order cumulant matrix $\mathbf{Q}_{u}$ are the terms $\left[\mathbf{Q}_{u}\right]_{K(k-1)+k, K(k-1)+k}=c_{k} \stackrel{\text { def }}{=} \int_{-1 / 2}^{+1 / 2} \int_{-1 / 2}^{+1 / 2} \rho_{k, k, k, k}\left(f, f^{\prime},-f^{\prime}\right) d f d f^{\prime}$. And the fourth-order cumulant term of (3.3) boils down to $\sum_{k=1}^{K} c_{k}\left(\mathbf{e}_{M}\left(\theta_{k}\right) \otimes_{c} \mathbf{e}_{M}\left(\theta_{k}\right)\right)\left(\mathbf{e}_{M}^{H}\left(\theta_{k}\right) \otimes_{c} \mathbf{e}_{M}^{H}\left(\theta_{k}\right)\right)$

\footnotetext{
${ }^{3}$ The following properties are proved for ULA, the proofs can be extended to URA along the same lines.
} 
where $\mathbf{e}_{M}\left(\theta_{k}\right) \stackrel{\text { def }}{=}\left(1, e^{i \theta_{k}}, e^{2 i \theta_{k}}, \ldots, e^{(M-1) i \theta_{k}}\right)^{T}$. The DOA algorithms alg(.) applied to $\mathbf{R}_{x}^{\text {to }}(n)$ define the mapping $\operatorname{alg}^{\text {to }}($.$) . This mapping satisfies condition 2 \mathrm{~b}$ of lemma 1 . Thus the second constraint upon $\mathbf{D}_{\Theta, \mathbf{R}_{x}}^{\text {alg }}$ of lemma 1 establishes the result.

2. In the single source case, the Toeplitzation is not sensitive to the temporal correlation of the sources. Proof: In this case, condition $2 \mathrm{a}$ of lemma 1 reduces to condition $2 \mathrm{~b}$. The expressions of $\mathbf{D}_{\Theta, \mathbf{R}_{x}}^{\mathrm{alg}} \mathbf{A}_{\mathrm{to}} \mathbf{C}_{R_{x}} \mathbf{A}_{\mathrm{to}} \mathbf{D}_{\Theta, \mathbf{R}_{x}}^{\mathrm{alg}}{ }^{H}$ coincide for the ARMA and harmonic sources with $\mathbf{D}_{\Theta, \mathbf{R}_{x}}^{\mathrm{alg}} \mathbf{A}_{\text {to }}\left(\sigma_{1}^{2} \mathbf{e}_{M}\left(\theta_{1}\right) \mathbf{e}_{M}^{H}\left(\theta_{1}\right) \otimes_{c} \sigma_{v}^{2} \mathbf{I}_{M}+\sigma_{v}^{2} \mathbf{I}_{M} \otimes_{c} \sigma_{1}^{2} \mathbf{e}_{M}\left(\theta_{1}\right) \mathbf{e}_{M}^{H}\left(\theta_{1}\right)+\sigma_{v}^{4} \mathbf{I}_{M^{2}}\right) \mathbf{A}_{\text {to }}\left(\mathbf{D}_{\Theta, \mathbf{R}_{x}}^{\text {alg }}\right)^{H}$.

3. In the case of several sources, the Toeplitzation is sensitive to the temporal correlation of the sources.

Proof: Because $\mathbf{e}_{M}\left(\theta_{k}\right) \mathbf{e}_{M}^{H}\left(\theta_{l}\right), k \neq l$ has not a Toeplitz structure, the column space of $\mathbf{A}_{\text {to }}\left(\mathbf{E}(\boldsymbol{\Theta}) \otimes_{c}\right.$ $\mathbf{E}(\boldsymbol{\Theta})$ ) does not belong to the column space of $\mathbf{E}(\boldsymbol{\Theta}) \otimes_{c} \mathbf{E}(\boldsymbol{\Theta})$. Condition 2a of lemma 1 is generally not satisfied. So the associated terms in (4.7) do not vanish, contrary to the single source case. Therefore the extra term

$$
\mathbf{A}_{\text {to }}\left(\sum_{1 \leq l \neq k \leq K}\left(\int_{-1 / 2}^{+1 / 2} S_{u_{k}}(f) S_{u_{l}}(f) d f\right) \mathbf{e}_{M}\left(\theta_{k}\right) \mathbf{e}_{M}^{H}\left(\theta_{k}\right) \otimes_{c} \mathbf{e}_{M}\left(\theta_{l}\right) \mathbf{e}_{M}^{H}\left(\theta_{l}\right)\right) \mathbf{A}_{\text {to }}
$$

that appears for ARMA sources, does not vanish in $\mathbf{C}_{\Theta}^{\text {to }}$. We note that the performance of the ARMA sources case and the harmonic source case coincides when the spectrums of the ARMA sources tend to be disjoint. At low SNR, $\mathbf{C}_{R_{x}}$ can be approximated by $\sigma_{v}^{4} \mathbf{I}$ and the Toeplitzation becomes insensitive to the temporal correlation of the sources. Furthermore the closed-form expressions given for two closely spaced sources at low SNR in ([11, rel. 9.118 and 9.119]) and in [12] remains valid for colored sources. At high SNR, the term (4.8) becomes dominant in $\mathbf{C}_{R_{x}}$ and the Toeplitzation becomes very sensitive to the temporal correlation of the sources, which will be confirmed in Section 5 . 


\subsection{Augmention techniques}

The linear or planar sparse arrays attract considerable attention as they lead to significantly improved performance [13] for spatially uncorrelated and white sources ${ }^{4}$. To show these techniques are sensitive to the temporal correlation of the sources, we consider only the standard method utilizing the direct augmentation approach [15]. To fix notations, consider a planar grid (a half wavelengh of the incident radiation equispaced). Let $\Gamma$ be the array characteristic function: $\Gamma(x, y)=\left\{\begin{array}{cl}1 & \text { if a sensor is in position }(x, y) \\ 0 & \text { elsewhere. }\end{array}\right.$. Let $\Lambda(d x, d y)$ be the autocorrelation function of the array characteristic function $\Gamma(\Lambda(d x, d y)$ represents the number of times the lag $(d x, d y)$ is present in the sparse array). $\mathbf{R}_{x}(n)$ and $\mathbf{R}_{x}^{\mathrm{au}}(n)$ denote the spatial covariance matrices associated with the fictitious $M_{1}^{\prime} \times M_{2}^{\prime}$-URA and the $M_{1} \times M_{2}$ augmented array respectively ${ }^{5}$. Then the direct augmentation approach [15] operates a linear transform on $\mathbf{R}_{x}(n){ }^{6}$ thanks to the augmentation operator $M_{1}^{2} M_{2}^{2} \times M_{1}^{\prime 2}{M_{2}^{\prime}}^{2}$ matrix $\mathbf{A}_{\mathrm{au}}:\left(\operatorname{Vec}\left(\mathbf{R}_{x}^{\mathrm{au}}(n)\right)=\mathbf{A}_{\mathrm{au}} \operatorname{Vec}\left(\mathbf{R}_{x}(n)\right)\right){ }^{7}$

$$
\begin{array}{r}
{\left[\mathbf{A}_{\mathrm{au}}\right]_{(h-1) M_{1}^{2} M_{2}+(j-1) M_{1} M_{2}+(g-1) M_{1}+i,(f-1) M^{\prime}{ }_{1}{ }_{1} M^{\prime}{ }_{2}+(l-1) M^{\prime}{ }_{1} M^{\prime}{ }_{2}+(e-1) M^{\prime}{ }_{1}+k}} \\
= \begin{cases}\frac{\Gamma(k-1, e-1) \Gamma(l-1, f-1)}{\Lambda(l-k, f-e)} & \text { if }\left\{\begin{array}{l}
l-k=j-i \\
f-e=h-g \\
0
\end{array}\right.\end{cases}
\end{array}
$$

Theorem 1 extends to $\mathbf{R}_{x}^{\mathrm{au}}(n)$ with the asymptotic covariance matrix $\mathbf{C}_{R_{x}}^{\mathrm{au}}=\mathbf{A}_{\mathrm{au}} \mathbf{C}_{R_{x}} \mathbf{A}_{\mathrm{au}}^{T}$. By the regularity condition (4.1) of subsection 4.1, the estimated DOA's are asymptotically normal with asymptotic covariance:

$$
\mathbf{C}_{\Theta}^{\mathrm{au}}=\mathbf{D}_{\Theta, \mathbf{R}_{x}}^{\mathrm{alg}} \mathbf{A}_{\mathrm{au}} \mathbf{C}_{R_{x}} \mathbf{A}_{\mathrm{au}}^{T}\left(\mathbf{D}_{\Theta, \mathbf{R}_{x}}^{\mathrm{alg}}\right)^{H}
$$

We note that lemma 1 does not apply to this situation because the mapping $\operatorname{alg}^{\mathrm{au}}($.$) is not defined on$ $\mathbf{R}_{x}(n)$, but on some terms of $\mathbf{R}_{x}(n)$ only. Consequently the insensitivity of the augmentation techniques

\footnotetext{
${ }^{4}$ An improvement of the performance of DOA algorithms when the sources are spatially correlated was proposed by using the redundancy averaging techniques. In these conditions, these techniques lead to asymptotical inconsistent and biased estimates [14].

${ }^{5} M_{1}=M_{1}^{\prime}$ and $M_{2}=M_{2}^{\prime}$ for restricted redundancy and $M_{1}<M_{1}^{\prime}$ and $M_{2}<M_{2}^{\prime}$ for unrestricted redundancy [13].

${ }^{6}$ We note that this linear transform is defined only for analysis purpose as $\mathbf{R}_{x}(n)$ is not observed.

${ }^{7}$ In case of linear arrays, $e=f=g=h=1$ and $(4.9)$ reads: $\left[\mathbf{A}_{\mathrm{au}}\right]_{(j-1) M+i,(l-1) M^{\prime}+k}=$ $\left\{\begin{array}{ll}\frac{\Gamma(k-1) \Gamma(l-1)}{\Lambda(l-k)} & \text { if } l-k=j-i \\ 0 & \text { elsewhere }\end{array}\right.$.
} 
to the distribution and the temporal correlation of the sources is not assured.

\section{Simulations}

We consider throughout this section, 2 sources of equal power $\left(\sigma_{k}^{2}\right)_{k=1,2}$, the SNR is defined as the ratio $\frac{\sigma_{1}^{2}+\sigma_{2}^{2}}{\sigma_{v}^{2}}$. The DOA's are estimated by the standard MUSIC algorithm and the number of Monte Carlo runs is 500 . These sources are issued from the DOA's $\theta_{k}=\pi \sin \theta_{k}^{\prime}$ with $\theta_{1}^{\prime}=30^{\circ}, \theta_{2}^{\prime}=20^{\circ}$ for the ULA and from the DOA's $\theta_{k}=\pi \sin \theta_{k}^{\prime} \sin \phi_{k}^{\prime}, \phi_{k}=\pi \cos \theta_{k}^{\prime} \sin \phi_{k}^{\prime}$ with $\theta_{1}^{\prime}=30^{\circ} \theta_{2}^{\prime}=20^{\circ}$ and $\phi_{1}^{\prime}=10^{\circ}$ $\phi_{2}^{\prime}=40^{\circ}$ for the URA.

The first experiment presents the case of two sources which are both spatially uncorrelated, white Gaussian, ARMA Gaussian (generated by a $(10,10)$ Butterworth filter driven by a white Gaussian noise) or harmonic. The centered frequencies of the ARMA and the frequencies of the harmonics are -0.25 and 0.25. Fig. 1 plots the theoretical MSE of $\theta_{k} \frac{1}{n}\left[\mathbf{C}_{\Theta}\right]_{k, k}$ and the estimated MSE E $\left\|\theta_{k}(n)-\theta_{k}\right\|_{\text {Fro }}^{2}$ as a function of the $S N R$ for an 10-ULA (the bandwidth is fixed to 0.5 for ARMA signals) after Toeplitzation. We observe that these estimated MSE's are in good agreement with the theoretical MSE's but are very sensitive to the temporal correlation of the sources. Fig.2 plots the theoretical MSE of $\theta_{k} \frac{1}{n}\left[\mathbf{C}_{\Theta}\right]_{k, k}$ for the ARMA Gaussian spatially uncorrelated sources as a function of the sources bandwidth for an 10-ULA. We observe that these theoretical MSE's increase with this bandwidth and begin increasing from the bandwidth 0.45 which is associated with the overlapping of the spectrum of the two sources. These MSE's increase from the value associated with two harmonic sources to the value associated with two white sources. The "saturation" phenomena observed in [10], desappears when the spectrum of the two sources are not overlapping. Note that the common expression of the MSE's obtained without Toeplitzation is close to the MSE's obtained after Toeplitzation for nonoverlapping spectra. Fig.3 plots the theoretical MSE of $\theta_{k} \frac{1}{n}\left[\mathbf{C}_{\Theta}\right]_{k, k}$ and the estimated MSE E $\left\|\theta_{k}(n)-\theta_{k}\right\|_{\text {Fro }}^{2}$ as a function of the number of sensors for an ULA for $S N R=20 d B$. We observe that these estimated MSE's are in good agreement with the theoretical MSE but these MSE's are very sensitive to the temporal correlation of the sources (the bandwidth is fixed to 0.5 for ARMA signals). These MSE's are decreasing with the number of sensors except for the case of overlapping spectrums where the "saturation" phenomena can lead to a degradation of the MSE. 
The second experiment presents the case of two sources with the same temporal parameters (for white source signals, the distribution is either Gaussian or discrete $\{-1,+1\})$ as in the first experiment but are impinging on a 12-Greene and Wood array [16] utilizing the direct augmentation approach [15]. Fig.4 plots the theoretical MSE and the estimated MSE of the angles $\theta_{k}$ and $\phi_{k}$ as a function of the SNR. The behavior of these MSE's is similar to those of the MSE's obtained for the ULA Toeplitzation situation.

\section{Conclusion}

In this paper, we have presented an asymptotic performance analysis of DOA finding algorithms using the stochastic model assumption in which both source and noise signals are possibly non-Gaussian and possibly temporally correlated. We have shown that the asymptotic statistical performance of the second-order DOA finding algorithms generally depend on the temporal correlation of the source and noise signals. But when the noise is supposed temporally uncorrelated, it is proved that the covariance-based DOA estimators that do not require the sources spatially uncorrelated are insensitive to the distribution and the temporal correlation of the source signals, unlike the Toeplitzation and the augmentation techniques that are very sensitive.

\section{References}

[1] B. Ottersten, Ljung, "Asymptotic results for sensor array processing," in Proc. IEEE Int. Conf. Acoust., Speech, Signal Processing (Glasgow, Scotland), pp. 2266-2269, March 1989.

[2] P. Stoica, A. Nehorai, "MUSIC, Maximum likelihood, and Cramer-Rao Bound," IEEE Trans. Acoustics Speech Signal Processing, vol. 37, no. 5, pp. 720-741, May 1989.

[3] P. Stoica, A. Nehorai, "Performance study of conditional and unconditional direction-of-arrival estimation," IEEE Trans. Acoustics Speech Signal Processing, vol. 38, no. 10, pp. 1783-1795, Oct. 1990.

[4] B. Ottersten, M. Viberg and T. Kailath, "Analysis of subspace fitting and ML techniques for parameter estimation from sensor array data," IEEE Trans. Signal Processing, vol. 40, no. 3, pp. 590-599, March 1992.

[5] J.F. Cardoso, E. Moulines, "A robustness property of DOA estimators based on covariance," IEEE Trans. Signal Processing, vol. 42, no. 11, pp. 3285-3287, Nov. 1994.

[6] R.J. Serfling, Approximation theorems of mathematical statistics, John Wiley and Sons, 1980.

[7] J.F. Cardoso, E. Moulines, "Asymptotic performance analysis of direction-finding algorithms based on fourth-order cumulants," IEEE Trans. Signal Processing, vol. 43, no. 1, pp. 214-224, Jan. 1995.

[8] P.J. Brockwell, R.A. Davis, Time series, theory and methods, Second Edition, Springer Verlag, 1991. 
[9] W. Xu, M. Kaveh, "The optimum weight of angle-dependent weighted MUSIC and its approximations," in Proc. Asilomar conf. on Signal, System and Computers, Nov. 1993.

[10] A. Gorokhov, Y. Abramovich and J.F. Böhme, "Unified analysis of DOA estimation algorithms for covariance matrix transforms," Signal Processing, Vol. 55, pp. 107-115, 1996.

[11] P. Forster, E. Villier, "Performances asymptotiques des méthodes HR," in S. Marcos editor, les méthodes à hautes résolution, Hermes 1998.

[12] P. Forster, E. Villier, "Simplified formulas for performance analysis of MUSIC and Min Norm," Proceedings of Ocean conf., Sept. 1998.

[13] S. Haykin, J.P. Reilly, V. Kezys and E. Vertatschitsch, "Some aspects of array signal processing," IEE Proccedings-F, Vol. 139, no. 1, pp. 1-26, Feb. 1992.

[14] K.C. Indukumar, V.U. Reddy, "A note on redundancy averaging," IEEE Trans. Signal Processing, vol. 40, no. 2, pp. 466-469, Feb. 1992.

[15] S.U. Pillai, F. Haber, "Statistical analysis of a high resolution spatial spectrum estimator utilizing an augmented covariance matrix," IEEE Trans. Acoustics Speech Signal Processing, vol. 35, no. 11, pp. 1517-1523, Nov. 1987.

[16] C.R. Greene, R.C. Wood, "Sparse array performance," J. Acoust. Soc. Am., Vol. 63, pp. 1866-1872, 1978. 


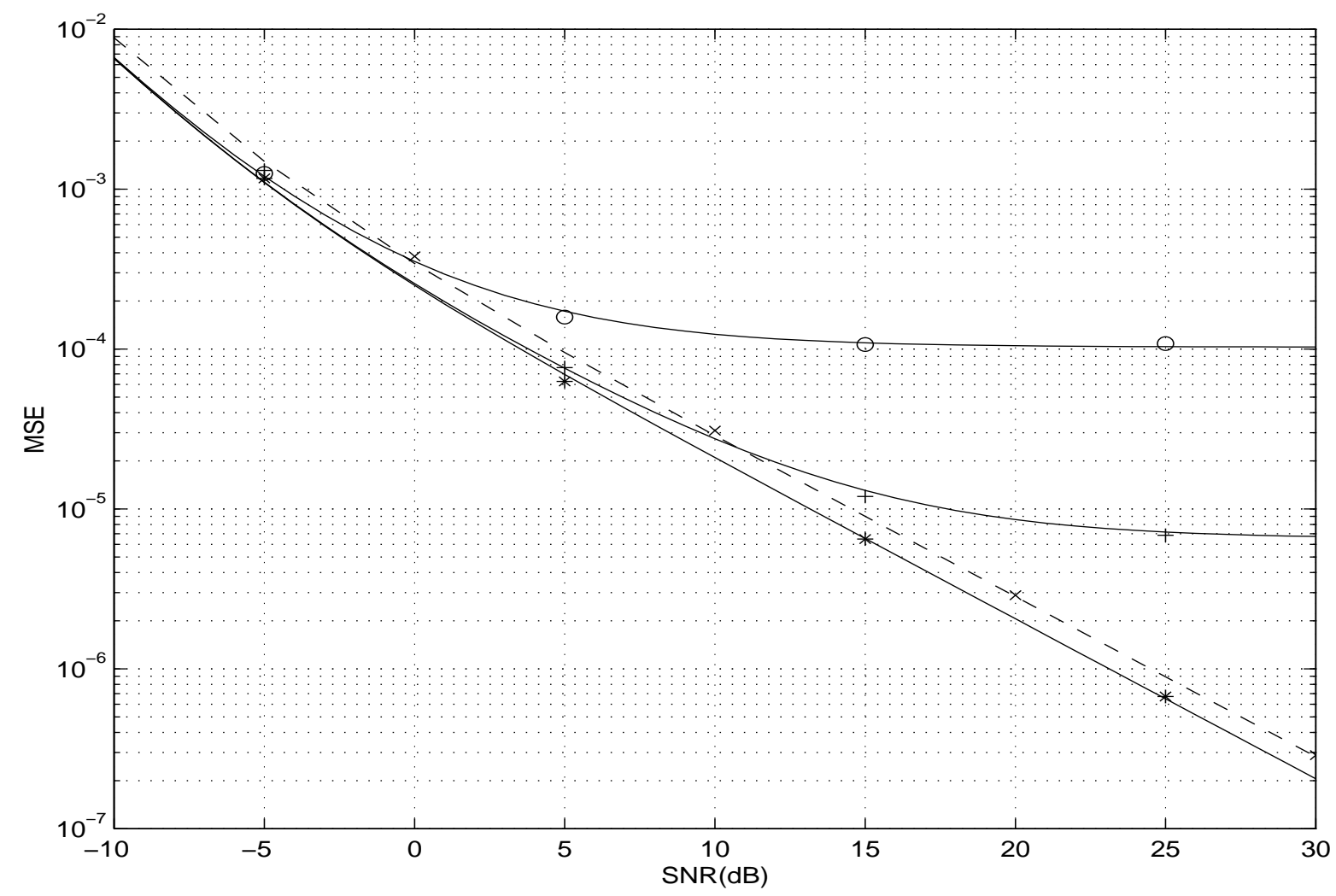

Fig.1 Theoretical and estimated MSE of $\theta_{k}(n)$ versus the SNR, for respectively white $(o)$, colored $(+)$ and harmonic $(*)$ signals for a 10-ULA array, $n=100$ after Toeplitzation $(-)$ and without Toeplitzation (- - -).

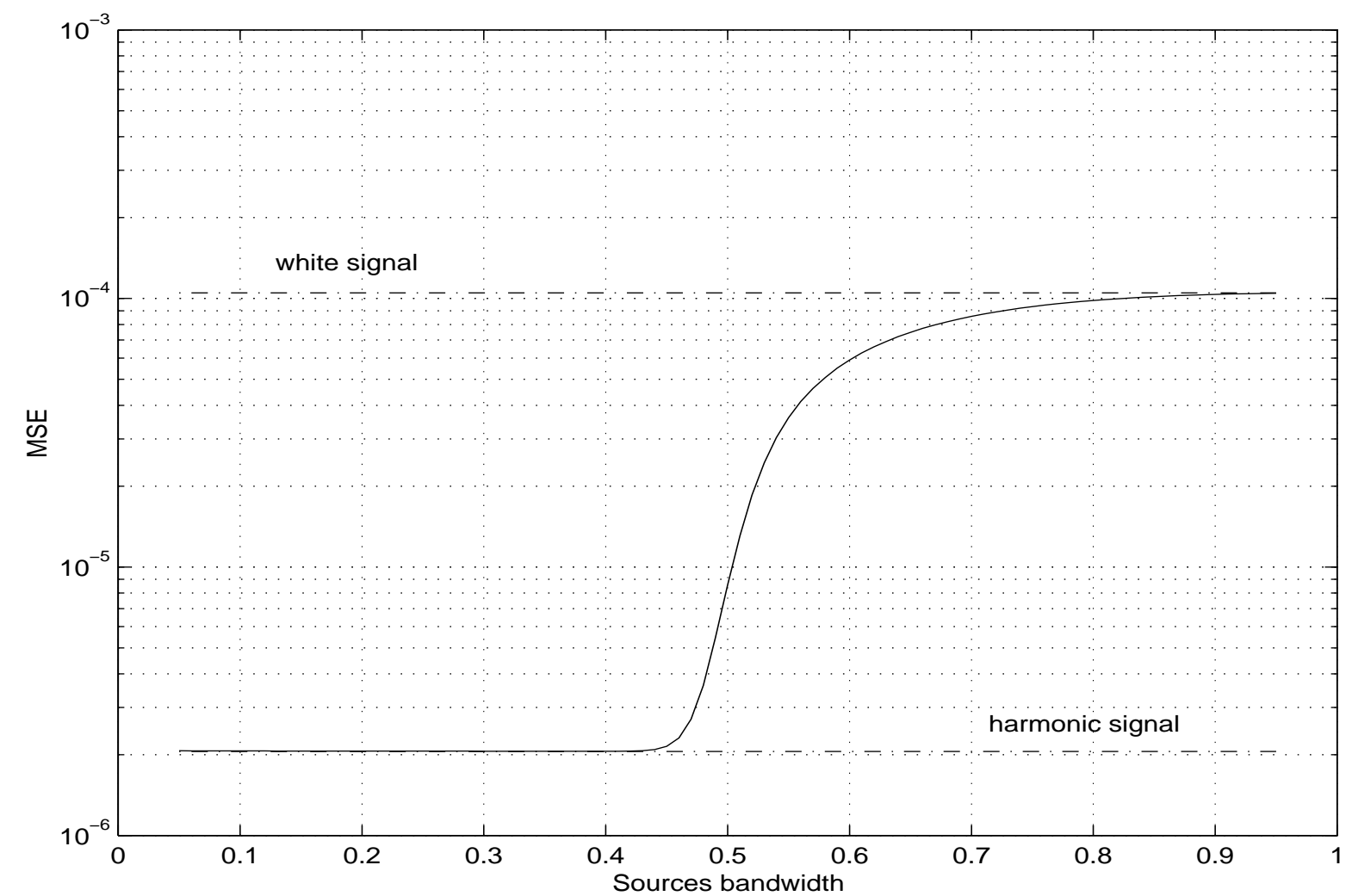

Fig.2 Theoretical MSE of $\theta_{k}(n)$ versus the sources bandwidth for a 10-ULA array, $S N R=20 d B$ after Toeplitzation. 


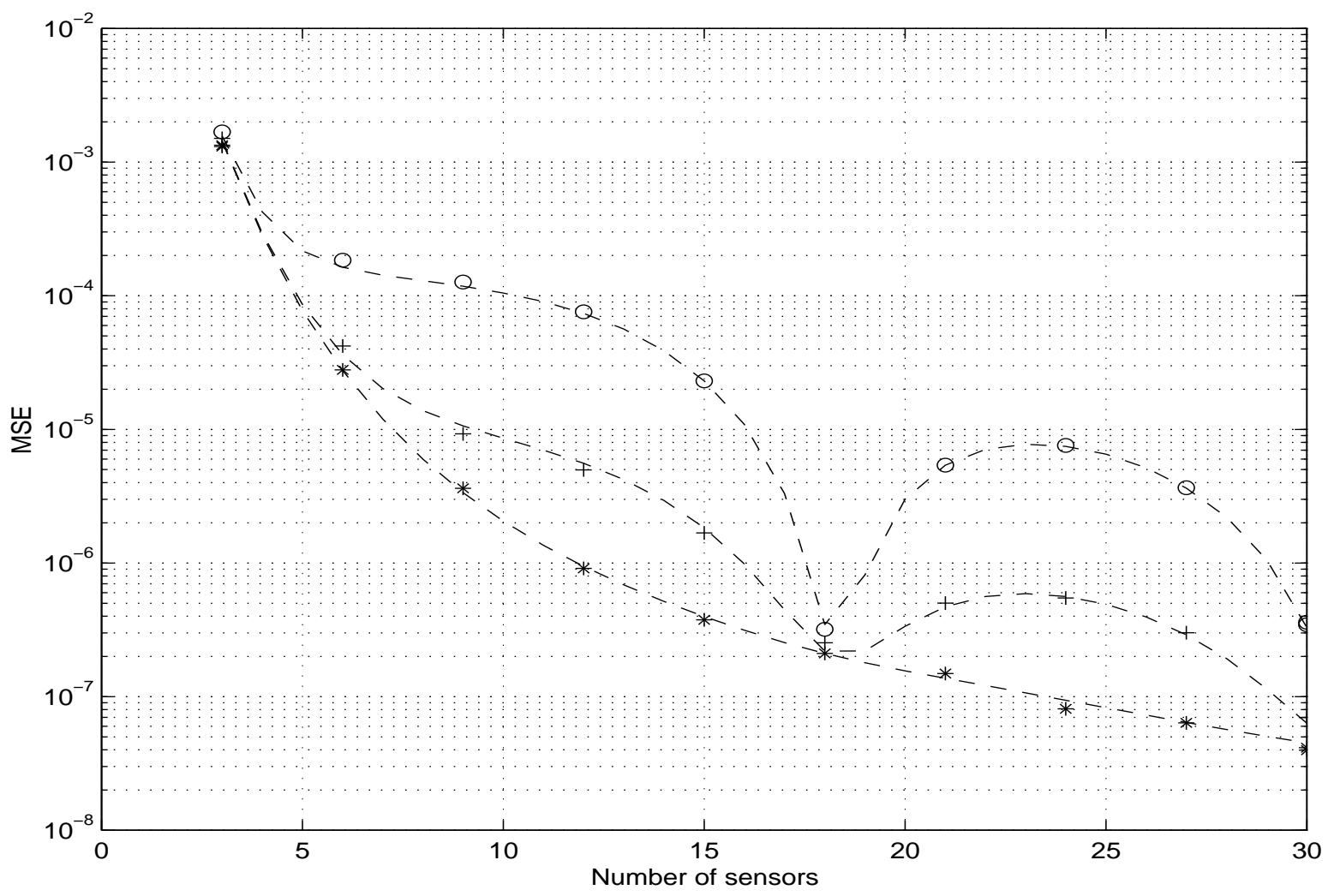

Fig.3 Theoretical and estimated MSE of $\theta_{k}(n)$ versus the number of sensors of an ULA array, $S N R=20 d B$, $n=100$ for white $(o)$, colored $(+)$ and harmonic $(*)$ signals after Toeplitzation.
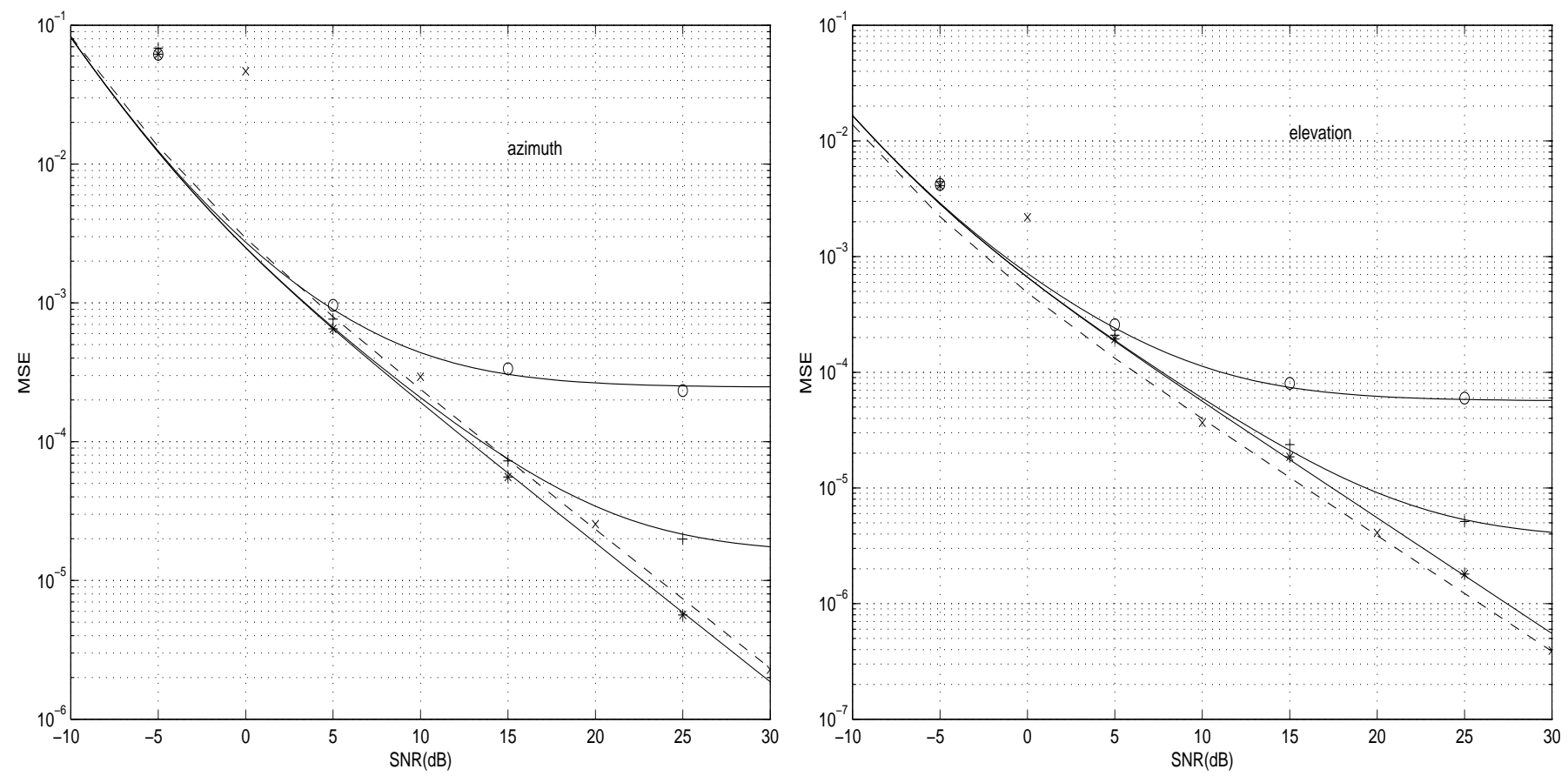

Fig.4 Theoretical and estimated MSE of $\theta_{k}(n)$ and $\phi_{k}(n)$ versus the SNR, for respectively white (either Gaussian or discrete) $(o)$, colored $(+)$ and harmonic $(*)$ signals for a $12-$ Greene and Wood array, $n=100$ after standard augmentation technique (-) and without augmentation (- - -). 